\title{
An Investigation of the Biological Properties of Organ- isms of the Pleuropneumonia Group, with Suggestions Regarding the Identification of Strains
}

\author{
BY D. G. FF. EDWARD \\ The Wellcome Veterinary Research Station, Frant, Sussex
}

SUMMARY: Seventeen representative strains of pleuropneumonia-like organisms were investigated to determine whether they could be identified by cultural methods.

The $L 1$ organism was readily identified by its morphology and by its cultural appearances on solid and in fluid and semi-solid media. Differences, depending on whether growth occurred anaerobically, whether growth was smooth or granular and whether a precipitate was formed in horse serum media, served to distinguish most of the other strains.

The requirements of serum and yeast extract for promoting growth and the effect of $\mathrm{pH}$ were studied with each strain.

Several strains fermented carbohydrates. Young cultures of some strains reduced methylene blue. Haemolysis around colonies in horse blood agar was noted with some strains; young broth cultures of these strains discoloured suspensions of horse erythrocytes. The factor discolouring erythrocytes was found in filtrates of old cultures of Asterococcus bovis.

Two strains were pathogenic for mice; when recovered after passage they showed no alteration in their nutritional requirements.

The organisms were resistant to penicillin; one strain grew in a medium containing 3000 units $/ \mathrm{ml}$.

In 1898 the filtrable agent causing contagious bovine pleuropneumonia was cultivated in a cell-free medium by Nocard \& Roux, and in 1910 the peculiar morphology of this organism was described by Bordet and by Borrel, DujardinBeaumetz, Jeantet \& Jouan. In 1923 agalactia in sheep was shown to be due to an organism of similar morphology. Within recent years a number of other similar organisms have been isolated. Some were obvious saprophytes, others were the cause of disease in animals; the pathogenicity of many, however, is still doubtful.

The group of pleuropneumonia-like organisms are characterized by their peculiar morphology and colonial appearances. Although capable of cultivation in artificial media, the smallest elements pass filters which will retain ordinary bacteria. These properties have distinguished them from bacteria. The group also includes similar organisms which have been isolated from cultures of bacteria. Although originally regarded by Klieneberger $(1936,1940)$ as symbionts, recent investigations suggest that they are variants of the bacteria. A classification and nomenclature for the group suggested by Sabin $(1941) a, b)$ has not been generally accepted, and would seem to be premature until the relationship of the group to the bacteria has been elucidated. There appear to be a number of species, identified by Sabin according to habitat and pathogenicity, each distinct antigenically. Until a nomenclature has been agreed the 
species and the existing strains must be referred to by the symbols given them when they were isolated.

Although the organisms causing bovine pleuropneumonia and agalactia are important in veterinary medicine, the other members of the group have aroused interest more as bacteriological curiosities. The recent isolation, however, of organisms of this group from human (Dienes, 1940; Dienes, Ropes, Smith, Madoff \& Bauer, 1948) and bovine (Edward, Hancock \& Hignett, 1947) genital tracts suggests that the group may acquire a greater importance. It has yet to be determined whether the organisms inhabiting the genital tract are pathogenic and the problem is made more difficult by a lack of knowledge regarding the general cultural and metabolic properties of the pleuropneumonia-like organisms. More than one species of pleuropneumonia-like organism may reside in the same situation. Sabin (1939) found several species with distinct serological and pathogenic properties inhabiting the respiratory tract of mice, and two different species were isolated from the genital tract of cattle (Edward, 1950). In the examination of material it is therefore necessary not only to determine that a pleuropneumonia-like organism is present but to identify the species.

Few attempts have been made to examine and compare a number of strains from different sources by the same methods. The most detailed observations were made by Klieneberger $(1938,1940)$, but they were largely confined to morphology, colonial appearances and antigenic constitution. Warren (1942) also compared the biochemical and metabolic activities of a number of strains. The present investigation was an attempt to distinguish between strains, representative of different species within the pleuropneumonia group, by examining those cultural properties which are commonly used in the identification of bacteria.

\section{TECHNICAL METHODS}

A total of seventeen strains were available for investigation; relevant details are given in Table 1. They were collected from laboratories in this country and in the U.S.A., and represent as many members as possible of the pleuropneumonia group. Unfortunately, cultures of several reported species are no longer in existence.

Strains were maintained by weekly subculture on plates of a medium made from ox-heart infusion broth, containing $1 \%$ peptone, to which was added $10 \%$ of a freshly prepared yeast extract and $20 \%$ of horse serum, the reaction

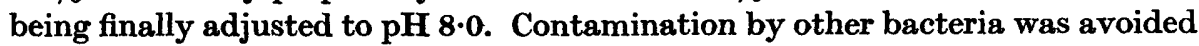
by the use of thallium acetate and penicillin (Edward, 1947a). The plates were incubated aerobically at $37^{\circ}$ for 3 days in closed tins containing exposed pieces of moist cotton-wool, thus ensuring a moist atmosphere throughout the period of incubation. Plates were inoculated by cutting out a small piece of agar bearing growth, which was then inverted over the plate to be inoculated and smeared across it.

For various investigations modifications, which will be described later, were made to this medium. A fluid medium (without agar) and a semi-solid medium (containing $0.3 \%$ agar), were also used. Two strains, L3 and L5, could not be 
Table 1. Details of strains investigated

Strain

Nos. 3278, 4159, 4782

Sewage $A$ and $B$

L 1 (Rat 30)

L3 (Ash)

L4 (Preston)

L5

M46

$\mathbf{C 1 4 0}$

H17

92, 93

B2

B30

B15

\section{Details}

Strains of the organism causing contagious bovine pleuropneumonia (Asterococcus bovis)

Saprophytic strains, serologically different, isolated from sewage*

Isolated from a culture of Streptobacillus moniliformis

Isolated from bronchiectatic lesions in a rat. Produced local abscesses in mice

Isolated from rats; produced abscesses and purulent arthritis. Has the pathogenic properties of L4, although its antigenicidentity with the original L4 strain has not been proved Isolated from mice; caused 'rolling disease'

Isolated from mice suffering from infectious catarrh

Isolated from mice; pathogenicity not known

From the human genital tract; isolated from a cervical swab

Isolated from the human genital tract

A P strain isolated from the bovine genital tract

A P strain from the bovine genital tract which had received only a few subcultures

An $\mathbf{S}$ strain isolated from the bovine genital tract
References

From The National Collection of Type Cultures

Laidlaw \& Elford (1936); received from $D r$ W. J. Elford

Klieneberger (1936, 1940); received from Dr E. Klieneberger-Nobel

Klieneberger \& Steabben (1940); received from Dr E. Klieneberger-Nobel

Klieneberger (1938, 1939);

Preston (1942); received

from Dr L. Dienes

Findlay et al. (1938); received from Dr E. Klieneberger-

Nobel

Edward (1947b)

Received from Dr L. Dienes

Isolated in this laboratory

Received from Mr S. R. M. Bushby

Edward (1950)

Edward (1950)

Edward (1950)

* The third serological type (sewage $C$ ), isolated from sewage, was unfortunately no longer available. The saprophytic strains isolated by Seiffert (1937) were closely related to sewage A (Klieneberger, 1940).

cultivated on the medium in routine use. They were inhibited by a $10 \%$ concentration of yeast extract and for their cultivation media containing $0.5-1 \%$ yeast extract were employed.

\section{RESULTS}

\section{Colonial appearances of surface cultures}

The surface colonies of organisms of the pleuropneumonia group are so characteristic that their examination with a dissecting microscope (magnification $\times 10$ ) provides the most convenient and reliable method for identifying an unknown organism as a member of the group, studies of its morphology in stained preparations and in unstained preparations examined by the dark-ground microscope affording confirmation.

The typical colony was hemispherical, sometimes slightly flattened, often with a central papilla or depression. When examined magnified $\times 10$ the most 
characteristic feature was a central spot, light brown in colour, produced by growth into the medium. The colony was translucent with a circular outline and the surface was either faintly marked with irregular lines or appeared finely pitted.

Colonies of the L1 organism, although having the same general characters, were quite distinctive. They were more opaque and were of a light brown colour, the central spots being a darker brown; their surface was coarsely marked with black lines. Therefore, although having the characteristic central spot, in some respects they resembled colonies of ordinary bacteria. $\mathrm{L} 1$ also gave a bacterial type of growth in fluid and semi-solid media. This strain was the only pleuropneumonia-like organism, isolated from a bacterial culture, which was available for examination; it would be interesting to determine whether other strains derived from bacterial cultures have cultural characters which are in part those of a pleuropneumonia-like organism and in part those of the parent bacterium, thus distinguishing them from the other members of the group.

Although colonies of all the other strains had the general appearance described, detailed examination revealed minor differences. For instance, strains differed in the maximum size attained by isolated colonies, and the relative size of the central spot, compared to the size of the whole colony, varied. There were also slight differences in the appearances of the central spots and in the manner in which they were demarcated from the rest of the colony. These differences sometimes sufficed to identify colonies of a particular strain. On the other hand, there were differences between the colonial appearances of the three strains of the one species, Asterococcus bovis. It is therefore unlikely that colonial appearances, which possibly also alter according to the conditions of cultivation, could be used to identify an unknown strain.

The production of certain characteristic effects by $\mathbf{P}$ strains, isolated from the bovine genital tract, after 5 days' growth on media enriched with horse serum has been described elsewhere (Edward, 1950). When viewed by the dissecting microscope numerous small black dots could be seen in the upper layer of the medium beneath and around the colonies, and there was often a crinkled film on the surface of the medium. Similar changes were produced by the strain L3 but not by any of the others. They are therefore important in the identification of the $\mathbf{P}$ and L3 organisms.

\section{Cultural appearances in fluid and semi-solid media}

Information of greater value for identifying members of the pleuropneumonia-group was obtained by examining cultures in a semi-solid medium (Beveridge, 1943). Some organisms grew as well anaerobically as aerobically, so that growth was equal throughout a tube of the medium; others only grew near the top where conditions were aerobic or micro-aerophilic. There were also differences depending on whether growth was smooth or granular.

The three strains of $A$. bovis only grew within $2 \mathrm{~cm}$. from the top of the medium. Colonies were fluffy and had poorly defined margins. When the inoculum was heavy, growth produced a generalized opacity in the upper part 
of the medium. Growth of the saprophytic strains (Sewage A and B, and B 15) was similar and also confined to the upper $2 \mathrm{~cm}$. of the medium. All P strains grew equally well throughout the medium, colonies being fluffy and diffuse with ill-defined margins. When the inoculum was heavy a generalized fluffy opacity developed in the medium, the opacity being greater than with most of the other organisms. The growth of the $\mathrm{L} 4$ organism was similar to that of the P strains, except that it was less opaque. When grown in a fluid medium, all the abovementioned strains produced a uniform opalescence, with or without a small deposit. The growth of these strains in both fluid and semi-solid media was thus predominantly smooth.

The other strains gave granular growths. Even when the inoculum was heavy the human genital strains (H.17, 92 and 93) grew in semi-solid media as small white circumscribed masses, which were dense and opaque, sometimes surrounded by a narrow fluffy halo. Colonies developed throughout the medium. Growth of the mouse strains, M46 and C140, was similar. The ability of M46, however, to grow anaerobically had only developed during artificial cultivation, as, when examined soon after isolation, it grew only near the surface (Edward, 1947 $b$ ). Strains L3 and L5 produced a similar type of growth which was confined to the upper part of the medium. In fluid media all these strains grew as a deposit of small dense white masses, the supernatant fluid remaining clear.

Cultures of the $L 1$ organism were quite distinctive. In the semi-solid medium colonies were found only near the surface and were relatively large, spherical and well-circumscribed; they were densely opaque and usually pigmented brown or black. In a fluid medium growth occurred as large spherical masses, densely opaque and often pigmented, which formed a deposit.

\section{Morphology}

The centrifuged deposits of horse-serum broth cultures were examined by dark-ground microscopy. Stained preparations of surface colonies were also examined. All strains exhibited the peculiar pleomorphic morphology characteristic of the pleuropneumonia group. The L 1 organism differed from the others. Under dark-ground illumination the majority of its elements were thicker and more refractile. Cultures of the $\mathbf{P}$ strains and of the L3 organism were also distinctive, because they contained peculiar spherical masses which could be stained by Giemsa and were highly refractile under dark-ground illumination (Edward, 1950). There were no significant differences between the appearances of the other strains to assist identification.

\section{Growth requirements}

Only the three saprophytic strains, B15 and Sewage A and B, grew at room temperature. These three strains also grew well on infusion agar base without the addition of yeast extract or serum. Enrichment with an animal protein, such as serum, was needed for the multiplication of all other strains; $10 \%$ of horse serum was the least amount which regularly gave maximal growth of all 
strains. Ox serum, rabbit serum and human ascitic fluid were compared with horse serum for their ability to promote growth. Most of the strains had been maintained for long periods on horse-serum media and grew best with horse serum, although some strains gave equally good growth with rabbit serum or ascitic fluid. Ox serum gave poor results with most strains. One strain, received from Dr L. Dienes, who had maintained it on ascitic fluid media, grew best with ascitic fluid and only poorly with horse serum. The results were interpreted as confirming the opinion of Sabin $(1941 a)$, who believed that during subculture organisms of the pleuropneumonia group become adapted to the protein of a particular species of animal. Thus, although strains differed in the way the various sera promoted their growth, the differences were probably properties of the strain itself, depending on how it had been maintained, and could not be used to differentiate between members of the group.

Incubation of plate cultures in a McIntosh \& Fildes' jar confirmed that anaerobic conditions prevented or impaired the growth of those strains which only grew near the surface of a semi-solid medium. An atmosphere of $10 \%$ carbon dioxide did not improve the growth of any strain; it impaired the growth of the three strains of $\boldsymbol{A}$. bovis, the three saprophytic organisms, the $\mathbf{P}$ strains and $L 1$, and completely inhibited $L_{1} 3$ and L 5 .

Effect of yeast extract. The addition of fresh yeast extract has been found to improve media used for cultivating this group of organisms (Edward, 1947a). The growths of all strains on horse-serum agar plates, containing 10, 5, 2, 1, $0.5 \%$ and no yeast extract were compared. Yeast extract did not improve the growth of the three saprophytic organisms, L1 and strain no. 3278 of $A$. bovis, but in a minimum concentration, varying between 10 and $1 \%$ effected a marked improvement in the growth of strains L 4, M46, C140, $\mathrm{B2}$ and $\mathrm{H} 17$.

Two strains, L 3 and L 5, were peculiar in being inhibited by a high concentration of yeast extract (Table 2). A smaller concentration, 0.5-1\%, enhanced the

Table 2. The effect of yeast extract on groroth; enhancement and inhibition (+, maximal growth; \pm , moderate growth only; Tr, slight growth only; -, no growth.)

\begin{tabular}{|c|c|c|c|c|c|}
\hline \multicolumn{6}{|c|}{ Yeast extract present (\%) } \\
\hline 0 & 0.5 & 1 & 2 & 5 & 10 \\
\hline \multicolumn{6}{|c|}{ Effect on growth } \\
\hline
\end{tabular}

Organism

$\mathbf{L 3}$

$\mathbf{L 5}$

$\mathbf{L} 4$

\begin{tabular}{|c|c|c|c|c|}
\hline \pm & + & + & \pm & $\mathbf{T r}$ \\
\hline+ & + & + & + & \pm \\
\hline $\operatorname{Tr}$ & \pm & \pm & + & + \\
\hline
\end{tabular}

* Included as an example of an organism, whose growth was not inhibited, but only enhanced by yeast extract.

growth of L3. The nature of the inhibiting factor was not ascertained, and it is not known whether it was identical with the factor promoting growth; neither factor was soluble in ether. It is probable that the sensitivity of these two strains developed during maintenance in culture. Both were strains recently received from Dr E. Klieneberger-Nobel and had been maintained by subculture for several years. When a subculture of the same strain of L5 was 
examined 2 years ago, its growth was then shown to be improved by addition of $10 \%$ yeast extract to the medium, there being no inhibition (Edward, $1947 a$ ).

Effect of $\mathrm{pH}$. The effect of $\mathrm{pH}$ was studied by adjusting the final reaction of the medium to values between $\mathrm{pH} 6.8$ and 9.2. The following strains were tested: $A$. bovis (3 strains), Sewage A and B, B 15, L 1, L3, L4, L 5, H17, B2 and M46. All except M46 and strain no. 4159 of $A$. bovis gave maximal growth in the range $\mathrm{pH} 7 \cdot 6-8 \cdot 4$. M 46 was slightly impaired by $\mathrm{pH} 7 \cdot 6$ and no. 4159 by pH 8.4. The results confirm the necessity for using as a routine a medium adjusted to $\mathrm{pH} 8 \cdot 0$. However, some of the strains gave maximal growth over a wide range of $\mathrm{pH}$. The strains of $A$. bovis and the $\mathrm{L} 1$ organism grew well at pH 6.8; growth of B2, L3 and L5 was not impaired at pH 8.8 and that of Sewage A not even at $\mathrm{pH} 9 \cdot 2$.

Fermentation of carbohydrates. A. bovis was shown to ferment certain carbohydrates, with the formation of acid only, by Tang, Wei, McWhirter \& Edgar (1935). Warren (1942) demonstrated the production of acid from glucose only by L 1, L3, L 4 and L5. Laidlaw \& Elford (1936) could not detect any fermentation of carbohydrates by the organisms they isolated from sewage. Similarly, Edward (1940) failed to demonstrate the production of acid from carbohydrates by an organism isolated from a transmissible pneumonia in mice. Apart from these observations there appears to have been no investigation of the fermentative properties of the pleuropneumonia group.

The organisms were grown on the surface of horse-serum agar plates, to which had been added $1 \%$ of carbohydrate and $0.005 \%$ of phenol red. Cresol red, also giving a change of colour between $\mathrm{pH} 7$ and 8 , had no advantage as an indicator. In a few confirmatory tests there appeared to be no advantage in carrying out the tests in fluid or semi-solid media. In all the tests control plates were included, containing phenol red but no added carbohydrate.

Altogether fourteen strains were examined and with eight the production of acid from certain carbohydrates was demonstrated (Table 3). The two sewage organisms fermented glucose, maltose, fructose, dextrin, starch and glycogen. Strain B15, an S strain from the bovine genital tract, gave similar reactions, except that acid was formed from galactose and not from fructose.

The $\mathbf{L} 1$ organism fermented glucose, maltose, fructose, galactose, mannose, dextrin, glycogen and salicin. Salicin, which is also fermented by Streptobacillus moniliformis (Topley \& Wilson, 1946), was not fermented by any other strain. Strains L3 and L5 fermented glucose, maltose, mannose, dextrin, starch and glycogen; it should be noted that mannose was not fermented by the saprophytic strains. One strain of Asterococcus bovis (no. 4159) behaved similarly to L3 and L5, except that there was also a weak fermentation of fructose. Another strain of this organism (no. 4732) did not ferment any carbohydrate; it is possible that its fermentative properties had been lost during long maintenance in culture. The third strain (no. 3278) gave weak and variable fermentations. Strains B2, H17, M46 and C140 and L4 did not ferment any carbohydrate. None of the strains fermented lactose, sucrose, mannitol, dulcitol, rhamnose, xylose, trehalose, raffinose, sorbitol, inulin and inositol. 


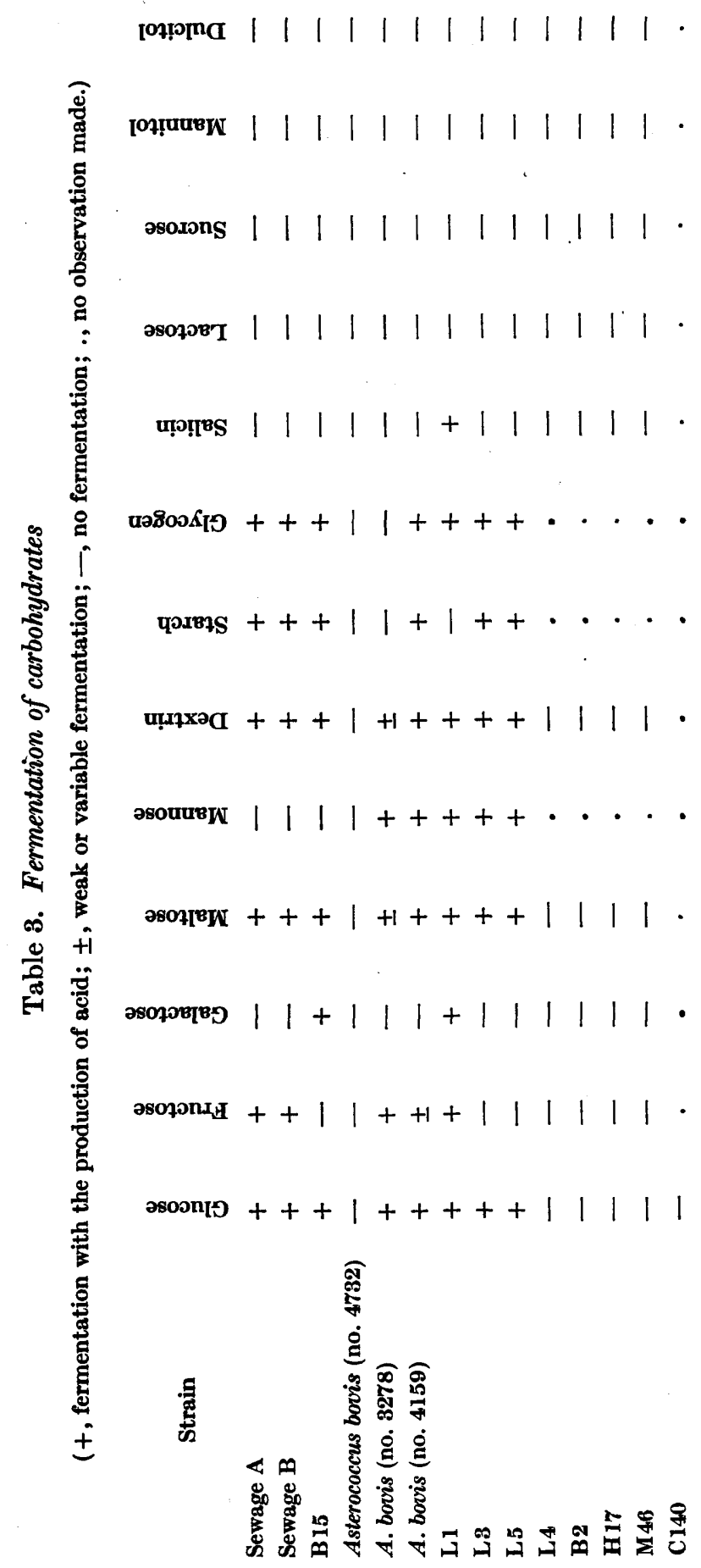




\section{Other metabolic characters}

Reduction of methylene blue. Holmes \& Pirie (1932) showed that cultures of $A$. bovis reduced methylene blue. Warren (1942) reported experiments which suggested that during repeated subculture of an $\mathrm{L} 4$ organism the ability to reduce methylene blue was lost in parallel with a decrease in virulence. Methylene-blue reduction tests were carried out on the strains under investigation by incubating, in $2 \frac{3}{4} \times \frac{3}{8}$ in. tubes at $37^{\circ}, 2 \mathrm{ml}$. of horse-serum broth cultures, to which had been added $0.4 \mathrm{ml}$. of a 1/10,000 solution of methylene blue. Cultures were examined each day after inoculation.

Reduction of methylene blue was demonstrated with the following strains: $A$. bovis (three strains), sewage organisms (two strains), B15, B2, L1, L3 and L5. No reduction was detected with $\mathrm{L} 4, \mathrm{H} 17, \mathrm{C} 140, \mathrm{M} 46$ (Table 4). Only young cultures reduced methylene blue, the greatest degree of reduction being noted when multiplication of the organism was maximal. This was particularly noticeable with the culture of B2. At the end of $24 \mathrm{hr}$. there was no apparent growth and the test was negative. At two days the medium was very faintly opalescent and the test was positive. By the third day the opacity of the medium had increased greatly but the test was then no longer positive. A positive methylene-blue test persisted longer in cultures of $\boldsymbol{A}$. bovis, becoming weakly positive or negative at the end of a week. This may be associated with a longer viability of this organism in broth cultures.

Haemolysis. Warren (1942) showed that filtrates of $A$. bovis 'reduced' haemoglobin; filtrates of other strains were inactive, although there was sometimes a green discoloration around colonies of L3 on a sheep-blood medium. Haemolysis produced by colonies of bovine $P$ and $S$ strains and of the sewage organisms was described by Edward (1950). In the present investigation strains were tested for haemolytic activity by two methods. First, haemolysis was noted around colonies on horse-blood agar. When pour plates were made on the organism was grown on the surface of this medium, it was not always possible to determine the result, owing to the discoloration which frequently occurred after incubation for several days. It was preferable to grow the culture on the surface of the ordinary horse-serum agar medium for 2 days and then to pour on top a thin layer of the same medium containing $5 \%$ of a horse red cell suspension. The results were read after incubation for a further 2 days. In the second method the strains were grown in horse-serum broth; each day 2 drops of a $5 \%$ suspension of horse erythrocytes were added to $1 \mathrm{ml}$. of culture. The mixtures were incubated at $37^{\circ}$ for $5 \mathrm{hr}$., being shaken at about half-hourly intervals and examined for changes in the colour of the erythrocytes.

On blood agar plates well-marked haemolysis was noted with the following strains: $\boldsymbol{A}$. bovis (three strains), sewage organisms (two strains), B 15, B2, L3, L4 and L 5. Strain C140 produced less intense haemolysis. Although there was slight clearing or discoloration round colonies of strains $H 17, M 46$ and $L 1$, there was no definite zone of haemolysis, such as was produced by the other strains.

When broth cultures of certain strains were incubated with horse erythro- 
320

D. G. ff. Edward

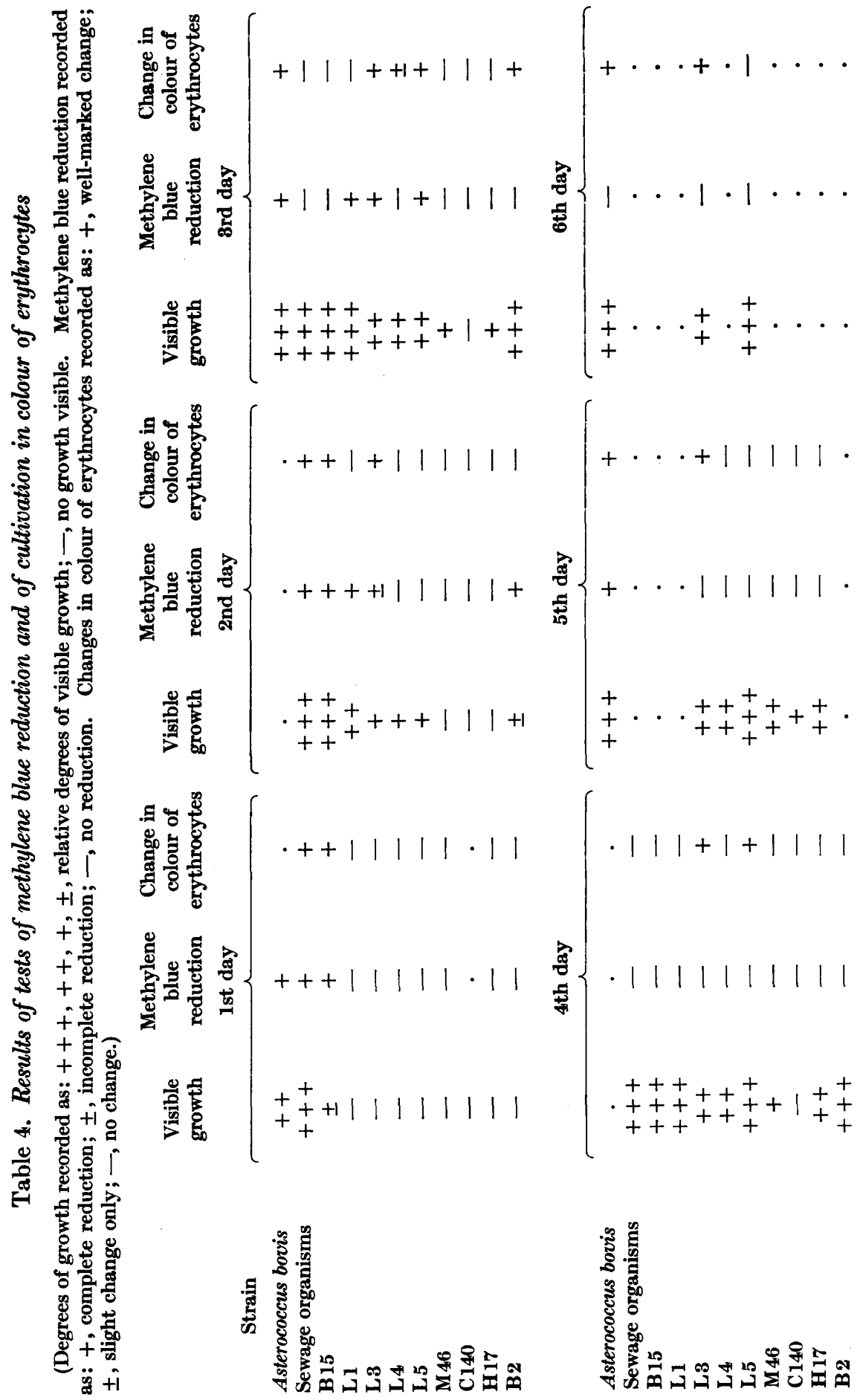


cytes, the suspension became a greenish brown colour after a few hours. There was no haemolysis; the cells, in fact, became more resistant and suffered no haemolysis even after $24 \mathrm{hr}$. incubation, whereas unaltered cells in control mixtures were haemolysed. The colour change was presumably due to formation of methaemoglobin and was not a simple reduction of haemoglobin. Greenish brown discoloration of erythrocytes was produced by $A$. bovis (three strains), sewage organisms (two strains), B15, B2, L3 and L5. Strain L4 caused slight discoloration only. Strains H17, M 46, C140 and L1 were inactive. The ability to discolour erythrocytes persisted in cultures of $A$. bovis and L3 for at least 6 days, but only young cultures of the other strains were active (Table 4). Reduction of methylene blue was also a property of young cultures only, but the two activities were caused by different enzymes. A few cultures reduced methylene blue but did not affect erythrocytes or vice versa.

Effect of centrifugation and filtration on the methylene-blue test and on 'haemolysis'. Experiments were carried out to determine whether the substances responsible for reducing methylene blue and affecting haemoglobin were associated with the organisms themselves or were liberated into the medium. Cultures of both sewage organisms after $24 \mathrm{hr}$. incubation were centrifuged for $60 \mathrm{~min}$. at full speed in a bench angle centrifuge. Although the original cultures reduced methylene blue rapidly and produced the characteristic change of colour with horse erythrocytes, the supernatants were without effect either on the methylene blue or on the erythrocytes. When resuspended in saline the sedimented organisms were strongly positive in both tests (Table 5). Cultures of $\boldsymbol{A}$. bovis, B2, L3 and L5 were similarly examined after 3 days' incubation at which time they reduced methylene blue and affected erythrocytes. The supernatants proved inactive in both tests. Therefore, with these organisms at this stage of growth, the enzymes responsible for reducing methylene blue and affecting haemoglobin were associated with the intact living cell.

Although with continued incubation cultures of the sewage organisms, L3 and B2 lost the ability to reduce methylene blue and to affect haemoglobin, 6-day cultures of $\boldsymbol{A}$. bovis and L3 still discoloured erythrocytes. The factor affecting erythrocytes was not present in the supernatant after centrifuging a 6-day culture of $\mathrm{L3}$. When, however, a similarly aged culture of $A$. bovis was centrifuged, the supernatant, although no longer capable of reducing methylene blue, still discoloured erythrocytes, the reaction being slower than with the original culture. The filtrate, obtained by filtering the supernatant through a gradocol membrane of $300 \mathrm{~m} \mu$. A.P.D. and shown to be sterile by failure to get a positive subculture from an inoculum of $1 \mathrm{ml}$., affected erythrocytes to the same degree as the original supernatant. It thus appears that although reduction of methylene blue is brought about by enzymes present in the intact organism, the substances affecting red cells are liberated from $A$. bovis during autolysis and can be demonstrated in filtrates. In this respect $A$. bovis behaves differently from the other pleuropneumonia-like organisms. 
322

\author{
D. G. ff. Edward
}

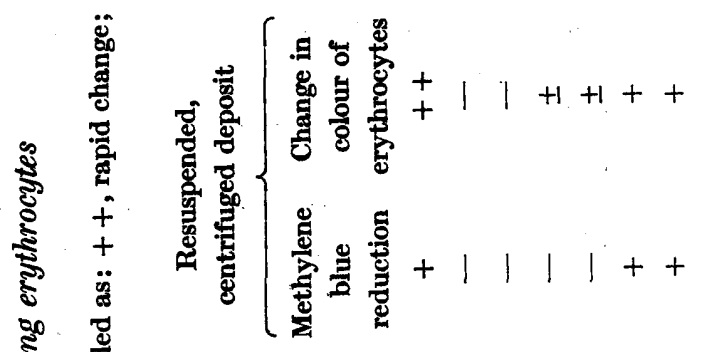

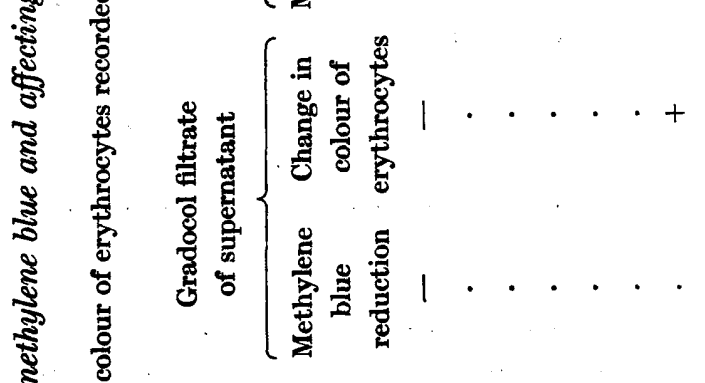

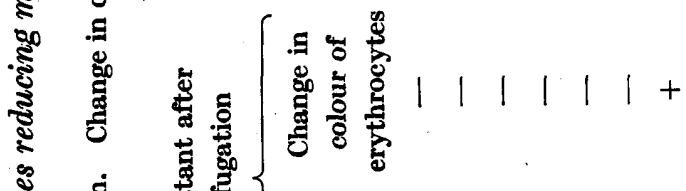

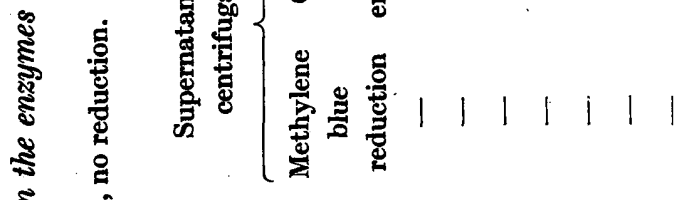

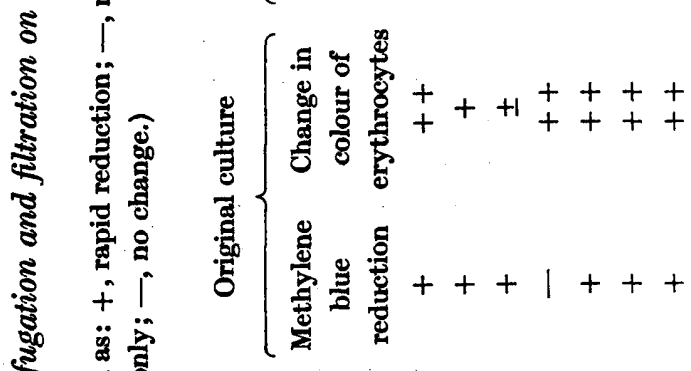

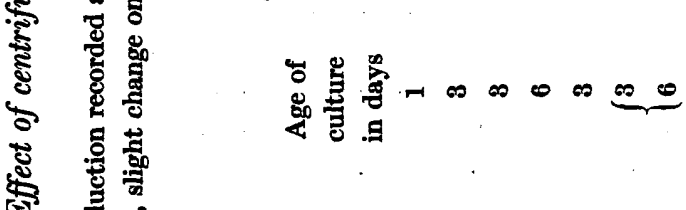

जी

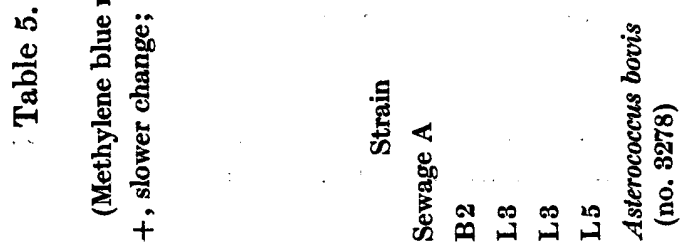




\section{Pathogenicity}

Only with a few strains was it possible to investigate pathogenicity in laboratory animals. The mouse strains L5, M46 and C140 were no longer pathogenic, but pathogenic effects were obtained with two strains, both originally isolated from rats. Cultures of the L3 organism in a semi-solid medium produced local abscesses when injected subcutaneously into mice. Serial subcutaneous passage was carried out, using pus as inoculum. The abscesses were small and localized to the site of inoculation; they soon discharged through the skin and the condition cleared up. The pus was without effect when inoculated intracerebrally into mice.

Cultures of the L4 organism were inoculated intravenously and intraperitoneally into rats; intra-abdominal abscesses resulted from the intraperitoneal injections and pus from these produced local abscess formation in mice when inoculated by the subcutaneous and intraperitoneal routes. The subcutaneous abscesses were more diffuse and caused greater induration of surrounding tissue than those due to L3. The intracerebral inoculation of mice with pus, or with cultures of the original organism, caused cerebral symptoms in about 3 days, followed by death, abscesses being formed in the brain. In no experiment did strain $L 4$ cause arthritis either in rats or mice. It appeared that the strain, although retaining its ability to cause abscesses, had lost much of its original virulence and no longer tended to localize in joints.

\section{Reinvestigation of strains after passage in animals}

No opportunity occurred to investigate strains immediately after their isolation. The L3 and L4, strains were therefore re-examined after recovery from animals, in order to find out whether their properties had been altered by animal passage. The $\mathbf{L} 4$ organism was recovered after four intracerebral passes in mice. At its first subculture growth was enhanced by yeast extract to the same degree as the original strain. Yeast, however, was not necessary for maximal growth when the infected brain suspension itself was plated, probably because of nutritional factors supplied by the inoculum.

The L3 organism was recovered from pus after three passes in mice. Both at the original plating of pus and at the first subculture the organism had the same nutritional requirements as the original strain. Growth of both was inhibited by a high concentration of yeast extract and enhanced by a smaller. Both grew well on horse-serum agar, but not on rabbit-serum or human ascitic-fluid agar. A few passages in animals had therefore not altered the peculiar nutritional requirements of this strain.

\section{Resistance to the bacteriostatic action of penicillin}

While using penicillin to obtain pure cultures of pleuropneumonia-like organisms from material contaminated with bacteria it became apparent that organisms of the pleuropneumonia group were extremely resistant and could resist concentrations which would inhibit the most resistant bacteria. Penicillin 
has also been used to isolate pleuropneumonia-like variants from certain bacteria by Dienes (1949) who demonstrated multiplication of a variant of this type in media containing $10,000 \mathrm{u}$. penicillin $/ \mathrm{ml}$. It was therefore of interest to determine the highest concentration of penicillin in which growth of a typical pleuropneumonia-like organism could occur.

Varying amounts of the calcium salt of penicillin (Burroughs Wellcome and Co. product for veterinary use, containing $20,000 \mathrm{u}$. per tablet) were added to a series of bottles of the semi-solid medium without thallium acetate, one bottle being left free from penicillin as a control. Each bottle was inoculated similarly with a culture of strain B2 and well shaken; a loopful was then immediately subcultured on a plate. After incubation for 24 and $48 \mathrm{hr}$. further subcultures were made with a loop. Thus the bottle with the highest concentration of penicillin, in which definite multiplication of the organism occurred, was determined. The concentration was found by assay, using the cup method, to be $3000 \mathrm{u} . / \mathrm{ml}$. at the time of inoculation and $2200 \mathrm{u} . / \mathrm{ml}$. after incubation for $48 \mathrm{hr}$. Twice this amount of the preparation of penicillin did not dissolve completely and prevented multiplication of the organism.

The inactivation of the penicillin during incubation was due to its lability and was not caused by the action of the organism. This was shown by assaying the penicillin in a bottle of the medium, which, after the addition of the same amount of penicillin, was incubated for $48 \mathrm{hr}$. without having been inoculated. The fall in concentration was not significantly different from that in the bottle in which $\mathrm{B2}$ was grown.

\section{IDENTIFICATION OF MEMBERS OF THE PLEUROPNEUMONIA GROUP}

In this investigation only stock strains, some maintained for many years in culture, were examined. It must be emphasized that there may be important differences in the properties of recently isolated strains. Moreover, only single strains of several organisms were examined. It is not certain that the properties are sufficiently constant to allow the identification of species within the pleuropneumonia group. The investigation, however, showed that most of the organisms studied could be identified by cultural properties. Only strains H17, M 46 and C140 could not be differentiated from each other. A scheme whereby a culture might be identified is shown in Fig. 1; it is based on the ability to grow at room temperature and on a serum-free medium, and on the appearances of surface colonies and of cultures in semi-solid and fluid media. The examination of other properties could be used for confirmation.

In Table 6, in which the findings are summarized, the strains are divided into three groups: (1) the saprophytic group; (2) the L1 organism, isolated from a culture of a bacterium; (3) strains isolated independently from animals. The saprophytic group were classified by Sabin (1941 $b)$ in a different family from the other organisms of the pleuropneumonia group. No major difference was found between the two strains of sewage organism, but the one $S$ strain from the bovine genital tract had slightly different fermentation reactions. Examination of a larger number of strains would be necessary to determine whether 


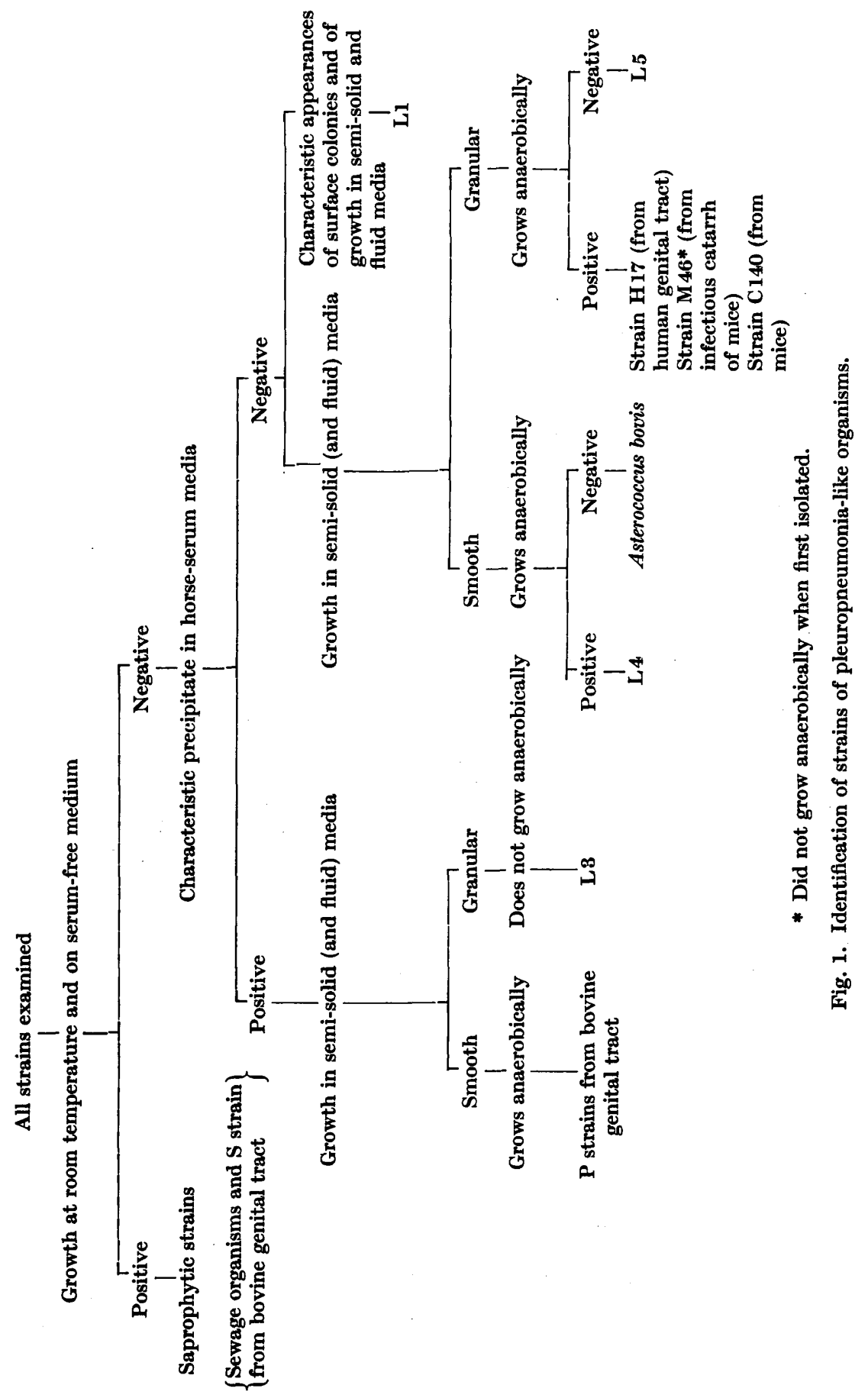


ริ)

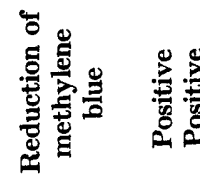

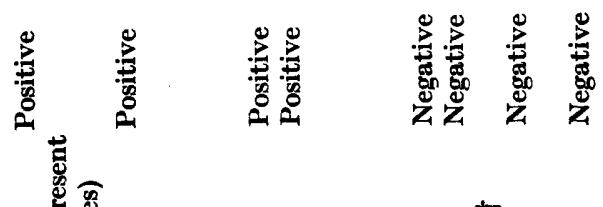

हूँ

罂

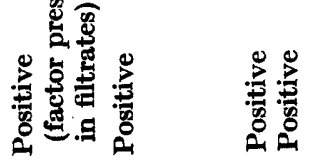

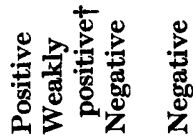

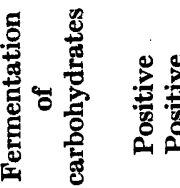

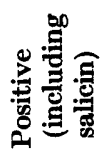

$\sum_{0}^{0}$

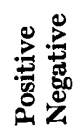

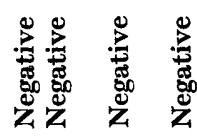

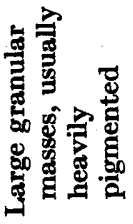

营 蛋

丞草

节䒿

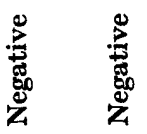

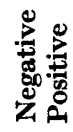

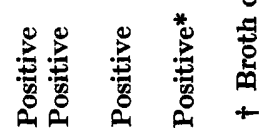

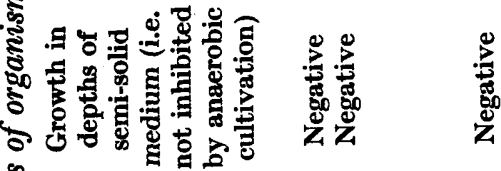

కू.

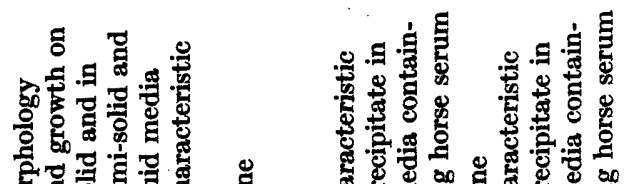

హ)

蛋惫

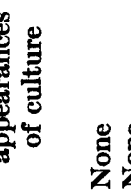

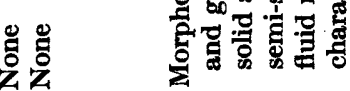

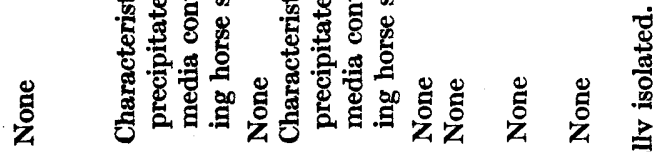

홀

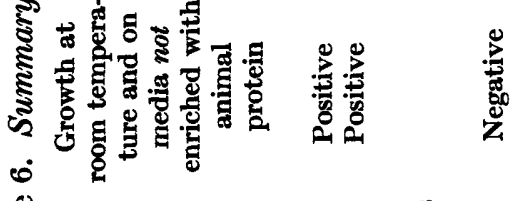

善

篦
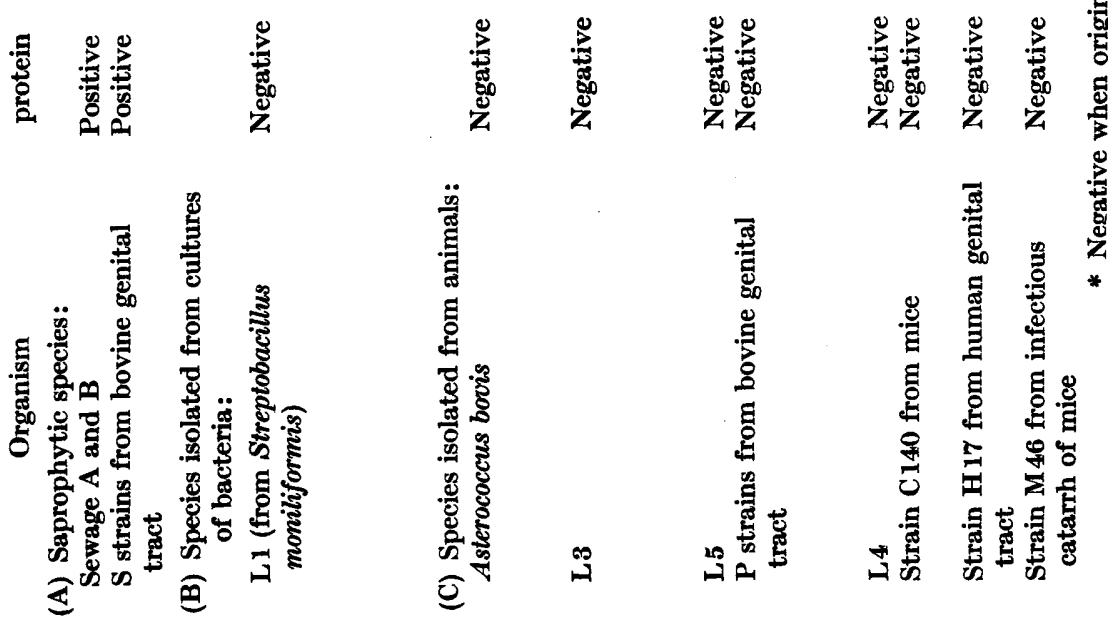

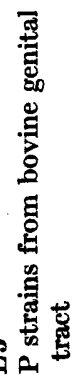

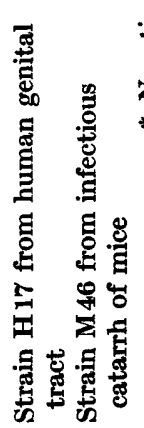


subdivision of the saprophytic group, according to cultural and biochemical properties, is possible. Organisms of the group are known to differ serologically (Laidlaw \& Elford, 1936; Klieneberger, 1940; Edward, 1950).

The $\mathrm{L} 1$ organism differed so much from the others in its morphology and colonial appearances that it could be easily recognized. The other organisms are listed in Table 6 with those with greatest biological activity appearing first. Data regarding $\mathrm{Ls}, \mathrm{L} 4$ and $\mathrm{L} 5$ were obtained by examining only single strains. Although only one bovine $\mathbf{P}$ strain was examined completely, all the strains isolated produced the characteristic changes in horse-serum media; they gave smooth growths and were not inhibited by anaerobiosis. Haemolysis in blood agar plates was noted with all the strains tested.

Only one strain from the human genital tract was examined in detail. It is possible that more than one member of the pleuropneumonia group may inhabit the human genital tract, each differing in its properties, and possibly also in pathogenicity. A detailed examination of many strains would be of interest, cultural properties being compared with antigenic constitution.

\section{DISCUSSION}

The pleuropneumonia-like organisms share certain distinguishing properties. Their smallest viable units are minute spherical bodies which can pass bacteriological filters; ultrafiltration studies of certain species suggest that they measure c. 150-200 $\mathrm{m} \mu$. From these are developed larger bodies, which possess only a delicate cell membrane and are particularly fragile, thus partly explaining the peculiar pleomorphism of these organisms (Smith, Hillier \& Mudd, 1948). In addition to reproduction by binary fission the large bodies multiply by multipolar germination. Pleuropneumonia-like organisms also produce characteristic colonies, due to growth into the medium. This probably depends upon a peculiarity in their metabolism. They are completely, or nearly completely, insensitive to the bacteriostatic action of penicillin.

The relationship of the pleuropneumonia group to the ordinary bacteria is complicated by the demonstration by Klieneberger(1936), Klieneberger-Nobel(1947) and Dienes(1949) that strains of pleuropneumonia-like organisms can beobtained from cultures of a number of bacteria. It now seems likely that these pleuropneumonia-like forms, or ' $L$ forms', are variants of the bacteria. The $L$ form differs from the original bacterium in its morphology, its smallest element being a particle which is filtrable, in its colonial appearance and in its resistance to penicillin. The last property facilitates its isolation in pure culture. The formation of a pleuropneumonia-like variant from Bacterioides funduliformis was studied under the electron microscope by Smith, Mudd \& Hillier (1948). The bacillus itself had morphological similarities to a pleuropneumonia-like organism; certain of its forms were extremely fragile and large round bodies developed which multiplied by multipolar germination. Cells of the pleuropneumonia-like variant differed in being smaller. It would seem that the essential differences, which distinguish the pleuropneumonia-like from other organisms, are, apart from size, related to their metabolism rather than to their morphology. 
Although pleuropneumonia-like variants of bacteria possess many characteristics typical of the pleuropneumonia group, many strains being stable with no tendency to revert to the bacillary form, it would seem desirable to classify them separately from the other members of the group. The L 1 organism included in this investigation was significantly different from the other strains morphologically and culturally.

The investigation of pleuropneumonia-like organisms has hitherto been hindered by technical difficulties. It must therefore be emphasized that the examinations described can be easily carried out, provided that certain precautions are taken. In the first place it is necessary to use an adequately rich medium. Secondly, contamination, which is particularly liable to occur when plates are incubated for several days in a moist atmosphere, should be controlled by using suitable bacteriostatic substances. Thirdly, certain modifications to technique, such as the methods for inoculating and incubating media, are advisable.

I wish to express my thanks to Dr E. Klieneberger-Nobel, Dr W. J. Elford, Dr L. Dienes and Mr S. R. F. Bushby for supplying me with cultures.

\section{REFERENCES}

Beveridge, W. I. B. (1943). Isolation of pleuropneumonia-like organisms from the male urethra. Med. J. Aust. $2,479$.

Bondet, J. (1910). La morphologie du microbe de la péripneumonie des bovides. Ann. Inst. Pasteur, 24, 161.

Borrel, A., Dujardin-Beaumetz, Jeantet \& Jouan (1910). Le microbe de la péripneumonie. Ann. Inst. Pasteur, 24, 168.

Dienes, L. (1940). Cultivation of pleuropneumonia-like organisms from female genital organs. Proc. Soc. exp. Biol., N.Y., 44, 468.

Dienes, L. (1949). The development of Proteus cultures in the presence of Penicillin. J. Bact. 57, 529.

Dienes, L., Ropes, M. W., Smith, W. E., Madoff, S. \& Bauer, W. (1948). The role of pleuropneumonia-like organisms in genito-urinary and joint diseases. Nere Engl. J. Med. 238, 509, 563.

EDWARD, D. G. FF. (1940). The occurrence in normal mice of pleuropneumonia-like organisms capable of causing pneumonia. J. Path. Bact. 50, 409.

EDWARD, D. G. FF. (1947 a). A selective medium for pleuropneumonia-like organisms. J. gen. Microbiol. 1, 238.

EDward, D. G. FF. (1947b). Catarrh of the upper respiratory tract in mice and its association with pleuropneumonia-like organisms. J. Path. Bact. 59, 209.

Edward, D. G. FF. (1950). An investigation of pleuropneumonia-like organisms isolated from the bovine genital tract. J. gen. Microbiol. 4, 4.

EDward, D. G. FF., Hancock, J. L. \& Hignetr, S. L. (1947). Isolation of pleuropneumonia-like organisms from the bovine genital tract. Vet. Rec. 59, 329.

Findlay, G. M., Klieneberger, E., MacCallum, F. O. \& Mackenzie, R. D. (1938). Rolling disease. New syndrome in mice associated with a pleuropneumonia-like organism. Lancet, ii, 1511.

Holmes, B. E. \& Pirie, A. (1932). Growth and metabolism of the bovine pleuropneumonia virus. Brit. J. exp. Path. 13, 364.

KLIENEBERGer, E. (1936). Further studies on Streptobacillus moniliformis and its symbiont. J. Path. Bact. 42, 587. 
Kuteneberger, E. (1938). Pleuropneumonia-like organisms of diverse provenance; some results of an enquiry into methods of differentiation. J. Hyg., Camb., 38, 458.

KuIENEBerger, E. (1939). Studies on pleuropneumonia-like organisms: the L4 organism as the cause of Woglom's 'pyogenic virus'. J. Hyg., Camb., 39, 260.

KIIENEBERGER, E. (1940). The pleuropneumonia-like organisms: further comparative studies and a descriptive account of recently discovered types. J. Hyg., Camb., 40, 204.

KLIENEBERgER-NoBeL, E. (1947). Isolation and maintenance of an L1-like culture from Fusiformis necrophorus (Syn. Bact. funduliforme. Bacteroides funduliformis). J. Hyg., Camb., 45, 407.

Kuteneberger, E. \& Steabben, D. B. (1940). On the association of the pleuropneumonia-like organism L3 with bronchiectatic lesions in rats. J. Hyg., Camb., 40, 223.

Laidlaw, P. P. \& Elford, W. J. (1936). A new group of filtrable organisms. Proc. Roy. Soc. B, 120, 292.

Nocard \& Roux (1898). Le microbe de la péripneumonie. Ann. Inst. Pasteur, 12, 240.

Preston, W. S. (1942). Arthritis in rats caused by pleuropneumonia-like microorganisms and the relationship of similar organisms to human rheumatism. J. infect. Dis. 70, 180.

SABIN, A. B. (1939). Mice as carriers of pathogenic pleuropneumonia-like microorganisms. Science, $90,18$.

SABIN, A. B. (1941 a). The filtrable micro-organisms of the pleuropneumonia group. Bact. Rev. 5, 1.

SABIN, A. B. (1941b). The filtrable micro-organisms of the pleuropneumonia group. (Appendix on classification and nomenclature.) Bact. Rev. 5, 331.

SEIFFERT, G. (1937). Ueber das Vorkommen filtrabler Mikroorganismen in der Natur und ihre Züchtbarkeit. Zbl. Bakt. (1 Abt., Orig.) 139, 337.

Smith, W. E., HiluIER, J. \& MoDd, S. (1948). Electron micrograph studies of two strains of pleuropneumonia-like (L) organisms of human derivation. J. Bact. 56, 589.

Smith, W. E., Mudd, S. \& Hulier, J. (1948). L type variation and bacterial reproduction by large bodies as seen in electron micrographic studies of Bacteroides funduliformis. J. Bact. 56, 603.

Tang, F. F., Wei, H., McWhirter, D. L. \& Edgar, J. (1935). An investigation of the causal agent of bovine pleuropneumonia. J. Path. Bact. 40, 391.

TOPLEY, W. W. C. \& WiLson, G. S. (1946). The principles of bacteriology and immunity, 3rd ed. Revised by Wilson, G. S. \& Miles, A. A. London.

WARren, J. (1942). Observations on some biological characteristics of the pleuropneumonia group. J. Bact. 43, 211.

(Received 3 November 1949) 